\title{
Foto-reporteros independientes en Internet, el rol de la red social y el de la comunidad virtual
}

\author{
Gabriela ORTIZ MiCHEL* \\ gortizmichel@iteso.mx
}

(Abstracts y palabras clave al final del artículo)

Enviado: 15 de febrero de 2013

Evaluado: 10 de marzo de 2013

Aceptado: 13 de marzo de 2013

\section{INTRODUCCION}

En el marco de la Sociedad de la Información uno de los temas relevantes es el de los cambios en la organización del trabajo. Manuel Castells (2000:295-296) explica que el modelo prevalente para el trabajo en la nueva economía es el de una "fuerza de trabajo base", reducida y que subcontrata según la necesidad y la demanda del mercado a una "fuerza de trabajo desechable". Esta última fuerza de trabajo, como Martín Carnoy (2002) menciona en su análisis sobre las tendencias en la flexibilización del trabajo, es dependiente de contratos temporales orientados a tareas y sin responsabilidad social compartida en las contrataciones. En lugar del trabajo centrado en una sola compañía, la tendencia es que las personas hagan su desarrollo profesional trabajando de manera independiente y por resultados (Wellman y Haythornthwaite y 2002:33). El profesional independiente tiene necesidad de contar con la habilidad para construir redes sociales que le permitan crear capital social para sobrevivir en este contexto inestable (obtener contratos, vender productos, relacionarse con proveedores, etc.). Más allá de la necesidad de éstas redes sociales, estos trabajadores no pertenecen a una única comunidad laboral en donde las ideas, las herramientas, las historias, las relaciones y el lenguaje no solo son compartidos, si no que constituyen la práctica misma (Wenger, McDermott y Zinder, 2002). Para Wenger $(2001: 125,132)$ la práctica es continua y es cambiante, y se construye como una historia compartida de aprendizajes. Aprender unos de otros es parte inherente de la práctica. Las prácticas evolucionan cuando las personas hacen juntas, negocian nuevos significados y aprenden unos de otros. El aprendizaje no es simplemente la adquisición de competencias, se trata de la formación de una identidad: participando en la práctica contribuimos a hacer que esa práctica sea lo que es. Hoy día para el trabajador independiente el contexto de la práctica está deslocalizado y multisituado, por lo que nos inquieta cómo se puede aprender en la práctica y cómo se puede, mediante el aprendi-

\footnotetext{
* Gabriela Ortiz es profesora titular de ITESO, en México.
} 
zaje continuo y compartido, ser co-desarrollador de una identidad en torno a la práctica misma, pero sin un contexto de referencia único.

Internet, la de la red de redes, ha ofrecido de manera abierta desde hace más de 30 años espacios para compartir, discutir, preguntar, aprender juntos. Hoy día, con la presencia de la red en la vida ordinaria de las personas, su estructura colaborativa web2.0 y el acceso a través de dispositivos móviles, se ha incrementado la aparición de espacios sociotécnicos que congregan a profesionistas. Es por ello que es de nuestro interés el estudio de estos espacios en tanto que pueden (o no) conformarse como espacios para extender las redes sociales profesionales, para aprender a lo largo de toda la vida, e incluso como espacios que favorecen la construcción y desarrollo de una práctica con significados e identidades compartidas, aunque desinstitucionalizada.

El trabajo que se presenta se desprende del estudio de un colectivo online orientado a fotorreporteros independientes, una práctica profesional multisituada y en donde el profesional tiene que aprender y hacer práctica dentro de un entorno muy amplio, pero sin el contexto inmediato de una institución. Este colectivo se estudió primero en 20067 cuando el colectivo tenía unos 2.5 años de vida e inicialmente unos 14 mil miembros. Se regresó a él en el 2011, 4 años después, cuando ya tenía más de 40 mil miembros. En este documento compartimos los resultados de las ob-servaciones, así como las reflexiones que se desprendieron de las formas diferenciadas en las que el espacio es usado y vivido por sus participantes, en relación con el aprendizaje continuado y el desarrollo desde ahí de una práctica situada y contextualizada fuera de las instituciones.

\section{EL ESTUDIO}

El colectivo que se estudió se llama Lightstalkers (www.lightstalkers.org) y está orientado a un profesional concreto, con una práctica de alguna manera caracterizada en las intenciones que se declaran en su página: "se desarrolló con el interés de respaldar los esfuerzos del profesional independiente relacionado con los medios, la investigación o los esfuerzos humanitarios, que por la índole de su trabajo viajan y muchas veces lo hacen a lugares inseguros o conflictivos". Lightstalkers fue concebido y desarrollado por un par de hermanos, uno fotorreportero independiente y el otro desarrollador Web. Con su proyecto buscaban atender las necesidades de la práctica del hermano fotorreportero y las de sus semejantes. El elemento clave considerado para este diseño fue la importancia de la red social, por lo que fue concebido como un espacio en que buscaban la participación, además de los semejantes, de la red ampliada (agencias de noticias, editores, impresores, abogados, activistas, fixers, traductores, etc.). La herramienta tecnológica ofrece un conjunto de recursos que consideraron acordes sus necesidades basándose para su diseño en las experiencias personales con otras herramientas sociales a fin de ofrecer características distintivas ${ }^{1}$.

Para su estudio partimos de la conceptualización de la tecnología como texto (Grint y Woolgar, en Hine, 2004:48-49), donde el proceso de diseño y desarrollo de

${ }^{1}$ Entrevista con el desarrollador, 2007 
la tecnología pasa por la generación de un imaginario de los usuarios y sus características, con base en un mayor o menor análisis de sus necesidades. Los desarrolladores escriben con ello un guión, y con él inscriben lo que ese usuario que imaginaron tendría que hacer, en nuestro caso con el software sociotécnico, para resolver esas necesidades que consideraron relevantes. A pesar de esto, el usuario, el usuario real, va a llegar con su bagaje personal, que puede no parecerse al de otro usuario real, y va leer ese texto y lo va a interpretar y, desde ahí, a usar, y con ello va a participar en la domesticación del espacio sociotécnico (Bakardjieva et al, 2001). Así, lo que puede producirse por el uso de una tecnología (prácticas, discursos, objetivos, problemas, etc.) no está prescrito por la tecnología, como tampoco por el usuario. Lo que puede producirse surge de la sumatoria del guión y de su interpretación, y en el caso de las tecnologías orientadas a la acción grupal, de la interacción entre los miembros y el artefacto. Es por ello que las metodologías cualitativas, y en especial la etnografía, se han vuelto muy populares para estudiar los colectivos en Internet. Las aproximaciones etnográficas favorecen la exploración de las complejas interrelaciones existentes entre los miembros de un colectivo y su relación con la tecnología misma (Hine, 2004).

La primera aproximación se realizó a través de un estudio etnográfico virtual (Markham, 1998), basado en una observación participante de 6 meses de finales del 2006 a mediados del 2007, complementada con entrevistas online (Ardèvol et al, 2003), una discusión sobre el significado de la participación en el colectivo abierta en su foro (Rezabek, 2000), el estudio y categorización de los hilos de discusión anteriores a esa fecha, así como la presencia del colectivo en Internet mediante búsquedas. En 2011 se regresa al colectivo, estudiando exclusivamente los foros. Se revisaron conversaciones correspondientes a un mes donde se replicó la categorización general de tipos de conversaciones que se encontró en 2006-7. También, a partir de una búsqueda, se escanearon todas las conversaciones entre 2007-2011 para identificar los hilos en los que hablan de sí mismos, en donde se encontraron tres muy relevantes para nuestro estudio.

\section{DESCRIPCIÓN DEL COLECTIVO}

Lightstalkers se desarrolló después de las experiencias que el hermano fotorreportero y sus colegas tuvieron en varios sitios de red social existentes en la red en aquella época, en donde se toparon con políticas que no les permitían atender cuestiones delicadas y que eran indispensables para su profesión, que implica viajar a regiones como zonas de guerra o escenarios de crisis humanitaria. De esas experiencias limitantes es que surge la idea de desarrollar un sitio de red social (bajo el concepto de Boyd y Ellison, 2007) especializado. Lo ofrecen gratuito y con reglas de convivencia muy sencillas en donde lo relevante es mantener un código de cooperación ${ }^{2}$.

\footnotetext{
2 Entrevista con el desarrollador, 2007
} 
La plataforma es muy sencilla: A nivel personal ofrece a cada usuario una página para su perfil. En ella se ofrecen recursos para que pueda ser usada como página profesional, como por ejemplo, datos de contacto, hacer referencia a sus publicaciones y presentar sus productos (galerías fotográficas). Buscando también atender la problemática del profesional en riesgo, ofrecen la posibilidad de registrar en su perfil los datos de ubicación, itinerarios de viaje, datos de emergencia (contactos, tipo de sangre, aeropuertos cercanos, etc.). A nivel del colectivo, Lightstalkers ofrece diversos recursos, como por ejemplo una sección para informarse mutuamente de sucesos globales de relevancia o para informar de sus publicaciones. El centro del movimiento es un foro de discusión, en donde cualquiera que sea miembro puede iniciar una conversación. Aunque existe una forma para que la persona que abre una conversación la categorice, son muy pocos los usuarios que lo hacen, volviendo poco útil esta estrategia de organización presente en el software.

Para arrancar el colectivo, que imaginaban también como una comunidad a desarrollar, los fundadores eligieron una estrategia: "cuidar la semilla con que se siembra la comunidad y el fomentar y mantener cierta consistencia general". Había que empezar invitando a las "personas correctas" (fotorreporteros con mucho respeto en la industria) y promover y mantener un diálogo online profesional entre ellos abierto para modelar la interacción que buscaban. ${ }^{3}$

En la etapa de estudio del 2007 se perfilaron los usuarios activos. El colectivo tenía entonces dos años y medio de vida. Se encontró que aunque hay muchas profesiones (la red ampliada) la mayoría de los participantes en los foros son reporteros o fotorreporteros. Ellos se consideran a sí mismos "viajeros" (travelers) con la misma o más fuerza que con la que definen su profesión. Por otro lado, se encontró que por sus características de profesionales independientes y de movilidad constante han sido usuarios asiduos de las tecnologías web, de las tecnologías móviles y, en especial los fotógrafos, de otro sinfín de tecnologías ligadas a los medios en que producen y dan a conocer su trabajo.

\section{LAS CONVERSACIONES}

El centro del movimiento en Lightstalkers se encuentra en las conversaciones en el foro. En el 2006-7 había el promedio unas 60 conversaciones activas diarias ( 180 mensuales). Este número bajó un poco en el 2011, a pesar del incremento en los miembros, a un promedio de 140 mensuales.

Se encontró que había, en lo general, dos tipos de conversaciones. Por un lado están las conversaciones que se vuelven relevantes para el trabajo cotidiano. Son conversaciones de apoyo e intercambios puntuales, donde se informan y se resuelve cuestiones concretas relacionadas con diversos aspectos de sus prácticas. Por otro lado están las conversaciones que podemos llamar "de largo aliento", en donde se puede discutir a profundidad temáticas de interés. Conversaciones que usan el foro

3 Entrevista con el desarrollador, 2007 
como un espacio comunitario en sí mismo, donde se tejen interrelaciones personales, significados compartidos, aprendizajes, donde también se juega, se inspira, y de alguna manera, se crea.

Creemos que estos dos tipos de conversaciones están relacionadas con formas construcción del espacio colectivo diferentes, que atienden de manera distinta las necesidades del profesional independiente, y que hablan de dos modos quizá complementarios de aprender y desarrollar profesión. Las primeras son reflejo de la red social en operación. Las de "largo aliento" creemos que tienen que ver con su desarrollo como comunidad, en donde se expresa una identidad compartida.

\section{LIGHTSTALKERS COMO RED SOCIAL}

It's a network that's global, truly global, and these are people that are doing essentially the same work that I'm doing. They're primarily photo-journalists, but they're in the field and doing the same kind of stuff. They're working with pictures. They're trying to find their own accommodations. So it's unbelievable that I can go on the Web site and say, "I'm going to the middle of nowhere and I need help," and have all these responses come in. ${ }^{4}$

Lightstalkers es un espacio de conexiones diversas: de gente con gente, con el trabajo de otros, con la industria, con los colegas, en fin, con la red social profesional y sus productos. En el primer tipo de conversaciones observadas se puede notar cómo estas conexiones se ponen en juego para resolver asuntos de la vida cotidiana de su quehacer profesional.

Entre las temáticas más comunes en el foro tenemos las cuestiones que giran en torno a los viajes. Este tipo de mensajes son normalmente peticiones concretas de ayuda o de información, que reciben respuestas concretas casi invariablemente. Se informan mutuamente sobre visados, servicios locales, hospedajes, dónde conseguir equipo para comprar o alquilar, servicios fotográficos, traductores, fixers y asistentes. También hay quien ofrece servicios, aunque en ese caso no es común encontrar respuestas. Otro tema igualmente común es el de la asesoría mutua en cuestiones técnicas, tanto de equipo fotográfico e informático, como de herramientas de software y de recursos web. Igualmente ponen a la venta su equipo. Es interesante notar que conversan mucho sobre las otras herramientas web que usan. Cuando discuten sobre otras herramientas, además de las cuestiones técnicas, se interesan también por los temas relacionados con el robo y fishing de sus fotografías, los tipos de seguridad que ofrecen los sitios, y las maneras de protegerlas desde la forma de subirlas a la web. Otra área muy común de conversación gira en torno a la práctica indepen-

\footnotetext{
${ }^{4}$ Es una red que es global, verdaderamente global, y estas son personas que están haciendo esencialmente el mismo trabajo que yo estoy haciendo. Son principalmente fotorreporteros, pero están en el campo y haciendo el mismo tipo de cosas. Están trabajando con fotos. Están intentando encontrar su propio hospedaje. Asi que es increible que puedo ir al sitio web y decir, "Voy al centro de ningúnlado y necesito ayuda", y tener todas esas respuestas. Entrevista referida en un hilo de discusión en Lightstalkers sobre sí mismos (http://www.lightstalkers.org/posts/ls-success-stories-)
} 
diente. Se discuten y ofrecen experiencias en torno a cómo anunciarse, cómo presentar sus portafolios en papel, o en sitios web o herramientas especializadas y se discute qué tan buenas o no son como espacio de promoción y/o venta. Se comparten las experiencias y relaciones con agencias, revistas, ONG. La parte económica tiene su lugar también, al consultarse sobre las formas de cobro, o cómo buscar una beca para obtener recursos para proyectos, o participar en un concurso o una convocatoria para formar parte de alguna agencia. Las cuestiones legales, también tienen su lugar, desde preguntas en torno al copyright hasta cómo pagar impuestos.

En esta categoría participan muchas personas diferentes ya que las solicitudes de información puntual son atendidas por el que tiene el conocimiento tácito. No es posible pues identificar una grupalidad única constante y presente en estas conversaciones.

Esas observaciones fueron complementadas con entrevistas y conversación sobre sí mismos en el sitio, en donde buscamos también los significados que Lightstalkers tenía para ellos. Entre ellas encontramos que en primer lugar los participantes encuentran especial valor en la red social en tanto que, como viajeros, suelen encontrarse en lugares que perciben lejanos y aislados:

As photographers, we tend to live very separate lives, live in odd places, work strange hours. Our paths don't cross often. Lightstalkers is a way of connecting with people [...] It's nice to know you're not alone, that someone you met at a riot in Ramallah is now living in Singapore, still chasing pictures. ${ }^{5}$

También encuentran valor en que les permite establecer conexiones concretas entre su trabajo y los posibles compradores de productos o servicios, entre su trabajo y su audiencia:

Yo tengo mi teléfono mi dirección todo está ahí, y eso funciona. Yo he trabajado con varias revistas bastante importantes de los Estados Unidos solo por estar aqui y tener mi perfil en LS. [...] Además LS es una manera de promoverse, claro. Yo gano una beca, la gente sabe [...] o tengo un multimedia, una película, y ellos lo ven. Asi que aunque por ejemplo un redactor no se interese, no quiera comprar lo que tengo, por lo menos lo ven. La audiencia ha crecido bastante por ser miembro de LS. ${ }^{6}$

Igualmente, para contactarse a nivel local:

Si vas a algún lado y necesitas contactar con alguien perteneciente a la red, puedes buscarlos en la lista de ciudades o países. La verdad es que es bastante útil y nos mantiene a todos en contacto y actualizados. ${ }^{7}$

${ }^{5}$ Como fotógrafos, tendemos a vivir vidas separadas, vivimos en lugares extraños, trabajamos en horarios extraños. Nuestros caminos no se cruzan con frecuencia. Lightstalkers es una manera de conectarnos con la gente [...] Es bueno saber que no estamos solos, que alguien que uno conoció en un motín en Ramallah ahora vive en Singapur, persiguiendo aún imágenes. Fotógrafo/Londres

${ }^{6}$ Entrevista con fotorreportero estadounidense en Santo Domingo. 
Aunado a las conexiones, los miembros dan mucho valor a la información que circula por Lightstalkers y de la cual son generadores.

I've got quite a good amount of useful practical info, and I guess I have given a few tips which were of some interest to some people. ${ }^{8}$

LS help me with so many tech issues iv lost count, it made airport $x$-rays a breeze and has helped me see how the photography/ media world works. ${ }^{9}$

Lightstalkers es percibido también como un espacio de aprendizaje, que trasciende el mero compartir información. Hablan de que el aprendizaje se da principalmente en los apoyos mutuos y desinteresados, así como en la posibilidad de ver el trabajo de los otros.

I check the profiles of the people who are there, and I often look as their posted portfolios. I see what people like to shoot, and their eye, their treatment of color, their cropping, etc. ${ }^{10}$

Por haberme juntado con LS, yo estoy mucho más avanzado y estoy acelerando mi educación. Yo sé que muchos jóvenes van a las escuelas para aprender fotoperiodismo, yo no hice eso, no me gusta eso. Yo creo que es algo más informal y LS es como una escuela que me ha enseñado mucho. ${ }^{11}$

Valoran también la calidad de la información que comparten, la consideran profesional, confiable y oportuna.

"I feel that for the most part I can trust the photographers here, and I can trust what they recommend". 12

En materia de confianza, hay que hacer notar que el hecho de ser identificables (los perfiles en Lightstalkers no son anónimos) depende no solo la posibilidad de las contrataciones o ventas, también la seguridad en viajes y, en general, la confianza en el informante sobre todo para temáticas críticas (Donath, 2003). Este no anonimato también juega un rol importante en las motivaciones para colaborar en el foro, ya que por ejemplo, según Kolock (2003), la posibilidad de identificar a los sujetos favorece la reciprocidad anticipada en colectivos online.

${ }^{7}$ Entrevista con fotógrafo mexicano freelance en Beijing.

${ }^{8}$ He obtenido una buena cantidad de información práctica útil, y creo que yo también he ofrecido algunos tipos que fueron de algún interés para algunas personas. Fotorreportero/Phnom Penh en, $\mathrm{http}: / / \mathrm{www}$. lightstalkres.org/whta_is_lightstalkers_for_you

${ }^{9}$ LS me ayuda con tantas cosas técnicas que ya perdí la cuenta, hizo que los rayos-x del aeropuerto fueran como una brisa, y me ha ayudado a ver cómo funciona el mundo de la fotografía/medios. Fotógrafo/Christchurch, Nueva Zelanda en, http://www.lightstalkers.org/posts/ls-success-stories-

${ }_{10}$ Reviso los perfiles de la gente que está ahi y siempre miro sus portafolios. Veo lo que la gente fotografia, y su ojo, el tratamiento del color, los recortes, que hacen, etc. Entrevista con abogado de medios y fotógrafo en Washington.

${ }^{11}$ Entrevista con estadounidense en Santo Domingo.

12 Siento que puedo confiar en los fotógrafos de aquí, y puedo confiar en lo que recomiendan. Fotógrafa/Boston, en http://www.lightstalkers.org/what_is_lightstalkers_for_you 
Aunque no lo nombran cuando se les pregunta por Lightstalkers, se observa que como red social también se movilizan alrededor de eventos como secuestros, arrestos o asesinatos de colegas que se encuentran en zonas o situaciones de riesgo, ya sea firmando peticiones, invitando a protestas, etc. Este activismo observado no podría hacerse al nivel global que logran convocar sin una mediación tecnológica (Rheingold, 2004).

Lo que les significaba en el 2007 se replica en una conversación que tuvieron en el 2008 sobre sí mismos. ${ }^{13}$ En el 2011 es observable que siguen fluyendo conversaciones y conexiones a través de necesidades puntuales, con respuestas prácticas, especialmente las del saber tácito que no existe en otro lado.

Como ya comentamos, estas son más bien micro-conversaciones de pocos con pocos, que surgen de la necesidad de uno que convoca a la red social. Son personas, organizaciones, productos y tecnología que mantienen un intercambio dinámico de información y apoyo respecto de necesidades y problemáticas puntuales y comunes, muy ligadas a su identidad profesional independiente. Podemos ver en el flujo de estos intercambios que la práctica está viva, que se implican temáticas siempre actuales sobre las que es necesario mantenerse informado y actualizado, aprendiendo. La red social que se pone en juego ante la pregunta o necesidad de uno, permite que el conocimiento tácito y puntual surja de la confluencia de las experiencias de algunos, de sus prácticas situadas, y que se exponga y quede inscrito en la red.

\section{LIGHTSTALKERS COMO COMUNIDAD}

\section{Friendship mostly and a supportive community of like minded people ${ }^{14}$}

Como mencionamos, formar una comunidad también fue una intención de los fundadores. No sabemos si lo que imaginaron es lo que ahora es, ya que lo que existe es una combinación entre el imaginario de los fundadores expresado en una herramienta sociotécnica y en su estrategia de semillarla/modelarla, con lo que buscan y necesitan los sujetos participantes, que está modelado por sus contextos externos y las negociaciones entre ellos. "El resultado es un establecimiento dinámico de significados sociales sistemáticos que capacita a los participantes para imaginarse como una comunidad" (Baym, 2004).

Para perfilar este colectivo como comunidad, y siguiendo a Baym (2010), usamos su caracterización de las 5 cualidades más relevantes para las comunidades en los contextos online: sentido del espacio, recursos y apoyos mutuos compartidos, práctica compartida, identidad compartida y relaciones interpersonales.

El sentido del espacio en una comunidad virtual no está meramente en el espacio sociotécnico y lo que aporta en términos de posibilidades y de fronteras para el

$13 \mathrm{http}: / /$ www.lightstalkers.org/posts/ls-success-stories-

14 Amistad por sobretodo y una comunidad de apoyo de personas afines. Fotógrafo/UK, en http://www.lightstalkers.org/posts/ls-success-stories- 
hacer. Está en el valor que como tal le dan las personas que participan del espacio. En Lightstalkers el sentido del espacio está presente cuando los miembros se refieren a ella como tal, cuando encontramos expresiones como "paso mucho tiempo aquí", "me gusta pasar el rato -hangout- con ustedes", "este lugar es increíble".

También es claro y presente el flujo de recursos y apoyos mutuos, como ya fue descrito cuando hablamos de la red social. Y aunque para Baym la práctica compartida se refiere al conjunto de rutinas que el colectivo tiene para la interrelación (que no estudiamos directamente), para nosotros, esa red social en acción también muestra un nivel de la práctica profesional compartida, en donde aprenden juntos a partir del conocimiento tácito que otros tienen para resolver el día a día.

Creemos que lo que hace que en este colectivo, además de operarse una red social, se pueda hablar de una comunidad, radica en la identidad compartida y en el tipo de relaciones interpersonales que de ella se desprenden y que al mismo tiempo, la conforman y que constituyen práctica en el sentido más profundo. Y esto lo podemos ver en la segunda categoría de conversaciones, las de "largo aliento".

Las conversaciones que llamamos "de largo aliento" están relacionadas en lo general con las problemáticas de la práctica, con sus significados y sus motivaciones, con la vida y con los sujetos mismos. Discuten sobre cuestiones éticas relacionadas con la profesión, la periodística y la fotográfica. También hablan de política, de la problemática de los medios y de la prensa libre. Hablan sobre arte, reciben y dan críticas sobre el trabajo de otros, hablan sobre cuestiones de salud, juegan y se divierten compartiendo fotografías por líneas temáticas o poemas o textos propios que dan pie a discusiones. También se enseñan mutuamente, se dan soporte, se indican recursos para el aprendizaje. A los participantes en este tipo de conversaciones, normalmente largas, les gusta debatir, argumentar y prepararse para sustentar sus ideas en las conversaciones.

Es en estas conversaciones donde descubrimos que los miembros iban perfilando una identidad compartida en el 2007. Estas conversaciones eran llevadas a cabo por un grupo pequeño y relativamente constante de personas. Era clara y notoria la presencia cotidiana y constante de algunas personas (hay que anotar que entre ellas se encontraban algunos de los miembros "semilla" y algunas figuras de mucho reconocimiento en el medio). Como grupo se reconocían, "bebían" juntos, se bromeaban, se daban consejos no pedidos y tenían un fuerte nivel de interacción interpersonal. También daban soporte a los novatos y participaban en los hilos más puntuales cuando conocían de la temática. No era un grupo separado y al margen de los demás, porque sus discusiones eran abiertas. Pero sí conformaban en 2007 una especie de élite ("las élites hacen que las cosas sucedan", Flichy, 2001) que iba dejando su huella respecto de quienes "son". Eran ellos, a través de sus discusiones, los que trascendían la identidad base meramente del "viajero independiente fotorreportero a zonas de riesgo", hacia una identidad que se vive apasionada por conocer mundos nuevos y darlos a conocer a otros, por contar historias, dar voz a los sin voz y por respaldar una prensa libre.

Para ellos ser viajero es también comunicación humana, conectarse con otros, aprender a leer sus mundos, es una fascinación, un modo de vida, un virus: 
Once you've been bitten by the travel bug it's hard to settle down. [...] the itch to go out there and see for myself, check things out with a mind as free and uncluttered as possible. ${ }^{15}$

Por sobre todo, les apasiona contar historias, y esto se vuelve su modo de vida. Optan por contar las historias de los sin voz, darle rostro a los sin rostro:

I take pictures of people so I can tell the story of people to other people. I don't focus on People but on people [...] the ones without voice or with very little of it ${ }^{16}$.

Tienen sus referentes claros respecto de quienes les inspiran:

"I managed to stop for a fraction of a second [at] this eternally fleeting life and show the image to others." -Kapuscinski ${ }^{17}$

Esta es una cita que ellos refieren y con la que se identifican en una de varias conversaciones ${ }^{18}$ en las que le dan tributo a Ryszard Kapuscinski "reportero visionario, uno de los grandes viajeros del siglo 20 y un fabulista" a quien reconocen como un "ancestro" reciente y un modelo. En esas conversaciones hablan de él como hablan de sí mismos expresando lo que les inspira en términos de lo que está en el fondo de su quehacer: viajar, aprender del mundo, aprehender el mundo, mostrarlo, contar historias y desde ahí, en la búsqueda de la verdad, optar por una prensa libre, que busca alternativas al nuevo poder de los medios de comunicación, lo cual incluso es descrito en una entrevista como la función del colectivo:

"Es para facilitar nuestros esfuerzos y también, si no para asegurar, por lo menos para apoyar a una prensa libre. Yo en realidad creo eso, yo creo que esa es una función de nuestro foro, que es muy importante para nosotros" 19

En cuanto a la dimensión de las relaciones interpersonales, encontramos expresiones como ésta cuando hablan de lo que es Lightstalkers para ellos:

Friends (real and virtual), discussion, information, challenges, headaches, projections (my whole projection project was born of what began here at LS), drunkeness,

15 Una vez que te picó el bicho del viaje es dificil arraigarse [...] la comezón por salir y ver por uno mismo, revisando las cosas con una mente tan abierta y limpia como sea posible. Entrevista en EightDiagrams (2007) http://wayneyang.wordpress.com/2007/03/03/john-vink-interview-part-ii/

16 Yo tomo fotos de gente para poder contar la historia de la gente a otra gente. Yo no me enfoco en la Gente si no en la gente [...] en los que no tiene voz o tienen poca voz. Entrevista con fotorreportero belga en Phnom Penh (2007)

17 "Fui capaz de detenerme por una fracción de segundo en esta vida eternamente fugaz y mostrar la imagen a otros"'- Kapuscinski

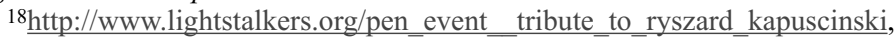

http://www.lightstalkers.org/ryscard kapuscinski cruzando fronteras y

http://www.lightstalkers.org/imperium by ryszard kapuscinski

19 Entrevista con estadounidense en Santo Domingo. 
arguments, life, vitality, exhibitions, information, lessons, ideas, advice, counteradvice, career-enhancement, career-damange, friends, time (lost and gained), insight, participation (in projections and shows), insight into not only photography but others ideas and lives and languages and cultures. ${ }^{20}$

En este tipo de expresiones ilustran que el colectivo va más allá de compartir información, de debatir y discutir los problemas de la práctica compartida: se encuentran entre amigos.

Las relaciones interpersonales, la exposición a los pensamientos y experiencias de otros a través de los diálogos profundos, del juego, del humor les permiten ser reflexivos respecto de sí mismos, de sus prácticas y de la vida misma.

You are able to read viewpoints on the same issue which I find quite helpful and refreshing even if I don't agree. ${ }^{21}$

In the end it is the benefit of getting outside of yourself in a bigger way than just talking to people on the street, at work or at a cocktail party, and sharing with them some interests and common concerns. ${ }^{22}$

It is about resituating things, people, what we do [...] I continually have to find out what my position is regarding the others, that I have to think about the consequences my actions have or don't have on the people around me. ${ }^{23}$

Esta oportunidad reflexiva sobre sí mismos y su profesión, junto con los significados que se tejían en las conversaciones de "largo aliento" son expresiones de la práctica activa de un modo específico, que puede o no ser compartida por otros profesionistas de la misma industria, pero que están tejidas y tejen, una identidad compartida.

Creemos que esta experiencia que recogimos en el 2007 habilitaba a Lightstalkers como un espacio que favorecía la construcción y desarrollo de una práctica con significados y aprendizajes compartidos fuera de una institución concreta. Ofrecía la posibilidad de desarrollar una práctica desde el hacer, negociar y

20 Amigos (reales y virtuales), discusión, información, retos, dolores de cabeza, proyecciones (mi proyecto entero de proyección nació de lo que comenzó aqui en LS), borracheras, argumentos, vida, vitalidad, exposiciones, información, aprendizajes, ideas, consejos, contra-consejos, mejora de mi profesión, empeora de mi profesión, amigos, tiempo (perdido y ganado), insight, participación (en proyectos y exposiciones), visión no solo de la fotografia, también de las ideas de otros y sus vidas e idiomas y culturas. Fotógrafo/Toronto, en http://www.lightstalkers.org/posts/ls-success-stories-

${ }^{21}$ Puedes leer puntos de vista sobre un mismo tema, que me parece muy útil y refrescante, aún cuando no esté de acuerdo. Fotógrafo/NY en http://www.lightstalkers.org/what is lightstalkers for you

22 Finalmente se trata del beneficio de salirte de ti mismo en una mañera más amplia que solo hablando con gente en la calle, en el trabajo o en una fiesta, y compartir con ellos algunos intereses y preocupaciones comunes. Abogado de medios y fotógrafo/ Washington en http://www.lightstalkers.org/what_is_lightstalkers_for_you

${ }_{23}^{3}$ Se trata de resituar las cosas, las personas, lo que hacemos [...] Continuamente tengo que encontrar cuál es mi posición respecto de los otros, tengo que pensar en las consecuencias que mis acciones tienen o no tienen en las personas a mi alrededor. Fotorreportero/Phnom Penh, http://www.lightstalkers.org/what is lightstalkers for you 
aprender unos de otros (Wenger, 2001), sin tener el contexto de una organización en donde la práctica sucede, e incluso con una perspectiva respecto de la industria con la que se relacionan.

Aprender en la práctica no solo implica participar en ella, no solo implica hacer. Aprender en la práctica y volverse competentes implica desarrollar la habilidad para conversar sobre la práctica y la de desarrollar una nueva identidad en referencia a los otros. Aprender implica pertenecer y posicionarse en un discurso (Gherardi y Nicolini, 2002). Lightstalkers ofrecía al fotorreportero independiente, y al que buscaba desarrollarse como tal, un espacio para vivir y desarrollar esta identidad colectiva en la práctica, para reflexionar en torno a ella y a sí mismo, y para posicionarse frente a un discurso.

\section{CAMBIOS EN LA COMUNIDAD}

Desde el perfil propuesto por Baym (2010) encontramos en el 2007 que Lightstalkers se había ido constituyendo en comunidad virtual a través de una práctica compartida, de una identidad negociada y negociándose, con sentido del espacio, con apoyos mutuos y con relaciones interpersonales que trascienden la mera práctica. Esta comunidad albergaba la posibilidad de un aprendizaje continuo a lo largo de toda la vida en el contexto de una práctica concreta y con una propuesta de un discurso sobre el ejercicio de esa la práctica, propuesto originalmente por una élite, que permitía este ir y venir entre aprender en la práctica y desarrollar la práctica misma negociando. Sin embargo, en la revisión del 2011 el tipo de conversaciones que llamamos "de largo aliento" que permitían eso se encontraron casi desaparecidas, reducidas básicamente a las lúdicas.

Aunque a principios del 2008 hay un hilo de conversación sobre lo que Lightstalkers les ha ofrecido a los miembros que contiene expresiones de mucho valor desde la perspectiva de la comunidad, para finales de ese mismo año aparece una llamada de atención. Se abre una conversación sobre la problemática que perciben en Lightstalkers en donde se menciona, por ejemplo, que en lugar de conversaciones serias ahora "se pegan videos musicales de Youtube" ${ }^{24}$. Es interesante notar la referencia necesitada a las personas que tejieron esa comunidad, esa que llamamos "élite":

A forum like this has a lot of new ideas and new members posting a lot of great stuff, but also has a lot of older, seasoned members who have some perspective on what $L S$ is all about. Unless the old bastards make a contribution and get momentum

\footnotetext{
24 En http://www.lightstalkers.org/posts/ls-manifesto

25 Un foro como este tiene muchas nuevas ideas y nuevos miembros publicando mucha s cosas geniales, pero también tiene muchos miembros antiguos, experimentados que tiene una perspectiva sobre de qué se trata LS. Al menos que los viejos bastardos hagan una contribución y generen un momentum que impulse la forma en la que ellos sienten que debe ir [...] esto no va a ir en ninguna dirección. Fotógrafo/Boston. En http://www.lightstalkers.org/posts/ls-manifesto
} 
going the way they feel it should go [...] then it's going to go along without any direction. 25

Tres años después de esa conversación, a finales del 2011 encontramos otra en donde se pregunta y discurren respecto de la baja en las conversaciones. Ahí encontramos un aporte de un miembro que ahora está en el Junta Directiva y que era participante activo de las conversaciones de "largo aliento":

There is a small group of founders and others trying to get help rebuilding a permanent infrastructure for those emerging/young photographers in the next (say, ten years younger) generation. It is proving difficult but there are glimmers which may yet bring LS into an even greater era, while still pursuing the same eleemosynary objectives that the original $L S$ went after ${ }^{26}$.

Este último texto muestra que la problemática que se venía esbozando desde hace tiempo tiene alguna identificación formal y que se están buscando soluciones. Pero lo más interesante de ese texto es ver que aparece un objetivo para estas acciones que no está descrito formalmente en ninguna declaratoria del sitio pero que la Junta Directiva reconoce como algo que esta comunidad aportaba: la oportunidad de modelar y dar soporte a la siguiente generación de fotorreporteros. Una intención que suponemos se enmarca en la identidad negociada y compartida originalmente del compromiso con la búsqueda por el desarrollo de una prensa libre.

\section{CONCLUSIONES}

En Lightstalkers encontramos una propuesta online que ha convocado por más de 7 años a la red ampliada de miles de fotorreporteros independientes que viajan. Pudimos observar que la función de Lightstalkers como red social, tan importante para los que hacen carrera en la profesión, no en una empresa, se pone en activo de manera continua favoreciendo que los miembros se conecten, atiendan necesidades, compartan saberes puntuales del día a día, aprendan juntos. Esta función, es muy relevante pero no es suficiente para constituir la práctica.

Las prácticas requieren un contexto y el contexto del profesional independiente no está abrigado por el paraguas de una empresa u organización, está disperso y deslocalizado. Lo interesante de Lightstalkers, en su función como comunidad, es que ofrece un contexto a través de una identidad compartida. Una identidad cargada de significados, que incluye una forma de verse, vivirse y situarse a sí mismos reflexivamente en sus pasiones y en la industria misma. Esta identidad ofrece un marco

${ }^{26}$ Hay un pequeño grupo de fundadores, que junto con otros están tratando de ayudar en la reconstrucción de una infraestructura permanente para aquellos fotógrafos emergentes/jóvenes en la siguiente (digamos, 10 años más jóvenes) generación. Está resultando difícil, pero hay destellos que puede traer LS a una aún mejor era, al tiempo que se siguen persiguiendo los mismos objetivos sobre los que LS se fundó. En http:/www.lightstalkers.org/posts/why-has-the-rate-of-new-posts-declined-social-media 
mucho más amplio y sobre todo más profundo para aprender, negociar y desarrollar la práctica misma, que el mero flujo de información que provee la red social.

Aunque encontramos al cierre del 2011 que Lightstalkers como comunidad se estaba desdibujando e incluso nos preguntamos si la función de red social seguirá operando igual de seguirse desvaneciendo la identidad, creemos nos ofrece pistas para pensar en nuevas formas de construir significados compartidos en torno prácticas independientes. Por ejemplo, arrancar la comunidad con "las personas correctas" promoviendo el diálogo online profesional entre ellos para modelar el tipo de interacciones que buscaban, nos parece que fue una forma de ofrecer una estructura social cargada de identidad, que favoreció la interacción entre los miembros. La invitación explícita a expertos reconocidos como semilla y a la industria y red social ampliada, posibilita, además del intercambio y conexiones, el desarrollo del conocimiento en torno a la práctica. A esto ayuda también el que todos los temas en torno a la práctica, tanto operativos, como de sentido, se permitan, y los más significativos hayan sido alimentados por varios años por una élite (o seasoned members como los llamaron).

La experiencia de Lightstalkers también nos sirve para identificar cuestiones que no se observaron y podrían favorecer más los entornos para el aprendizaje y desarrollo de conocimiento continuos que requieren las prácticas. Por ejemplo, aunque podemos observar que generan, comparten y mantienen información de muchos niveles, no se observaron estrategias ni operaciones que les permita capitalizar ese conocimiento de manera cosificada. La acumulación de la experiencia se da a nivel personal, no colectivo, y aunque online todo queda inscrito, la falta de herramientas apropiadas de búsqueda y de organización adecuada de sus propias conversaciones favorece que la información que genera se volatilice y se repita continuamente.

Ver que existe la posibilidad del aprendizaje y desarrollo en la práctica a través de comunidades virtuales de profesionales independientes, nos remonta al aprendizaje situado en las prácticas, en donde desarrollar una identidad como un miembro de una comunidad y convertirse en alguien competente, son parte del mismo proceso (Lave, 1991). Colectivos como Lightstalkers están mostrando que es posible desarrollar estas comunidades de práctica fuera del esquema de una organización (o de una escuela) y también fuera del esquema de una práctica local tradicional. Creemos que las pistas están en el modo mismo de la red Internet: son formas desinstitucionalizadas, abiertas, multisituadas, colaborativas. El aprendizaje y el desarrollo de la práctica parte del "enredarse" con otros más allá de solo lo local; se trata de ver conexiones entre campos, ideas y personas, así como de compartir el saber propio, el saber tácito, por muy pequeño o muy grande que sea. Se trata de abrir el conocimiento, de discutir la práctica, de reflexionar en torno a ella, de verse a uno mismo y verse en la práctica. En modo red se ofrece la posibilidad aprender mediante la participación en un discurso y el posicionarse respecto de éste, co-transformando la práctica misma desde ahí.

Bajo los nuevos esquemas de la Sociedad de la Información, en donde la práctica independiente crece rápidamente, especialmente en ciertas profesiones, es muy interesante encontrar espacios gratuitos, gestados y desarrollados por un grupo que encuentran valor en construir comunidades de práctica desinstitucionalizadas y mul- 
tisituadas en donde se pretenda acoger y ofrecer espacios para el desarrollo de las siguientes generaciones. Para nuestro caso, sería necesario mantener una investigación longitudinal para observar cómo la búsqueda por restaurar la comunidad con un objetivo específico se pone en juego, o no, y cuáles son las implicaciones de esto en el proceso del colectivo vivo.

\section{REFERENCIAS BIBLIOGRÁFICAS}

Ardévol, Elisenda; Bertrán, Marta; Callén, Blanca; Pérez, Carmen. "Etnografía virtualizada: la observación participante y la entrevista semiestructurada en línea". Athenea digital. 3, 2003, pg72-92. DOI:(Revista) ISSN 1578-8946 [última consulta: 11/11/2012] http://psicologiasocial.uab.es/athenea/index.php/atheneaDigital/article/view/67

BAKARDJIEVA, Maria y Smith, Richard. "The internet in everyday life. Computer networking from the standpoint of the domestic user", en New Media \& Society. Vol3(1):67-93. SAGE Publications, 2002.

BAYM, Nancy. "La emergencia de la comunidad on-line". En: Jones, S.G. (editor) Ciebersociedad 2.0, Barcelona, Editorial UOC, 2004.

BAYM, Nancy. Personal connections in the digital age. Cambridge, Polity Press, 2010.

BoyD, d. m., y Ellison, N. B. "Social network sites: Definition, history, and scholarship". Journal of Computer-Mediated Communication, 13(1), 11, 2007. DOI: 10.1111/j.1083-6101.2007.00393.x. [última consulta: 11/11/2012] http://jemc.indiana.edu/vol13/issue1/boyd.ellison.html

CARNOY, Martin. Sustaining the New Economy. Work, Family, and Community in the Information Age. New York: Russell Sage Foundation / Harvard University Press, 2002.

CAstells, Manuel. The Rise of the Network Society. Malden: Backwell Publishers Ltd., 2000.

Donath, Judith S. "Identidad y engaño en la comunidad virtual". En: Smith y Kollock (editores) Comunidades en el ciberspacio, Barcelona, Editorial UOC, 2003.

FLICHY, Patrice. "Internet. The social construction of a network ideology", en The Social Sustainability of Technological Networks. University of New York. Warner Graduate School of Public Service. Abril 2001, pg17-21

GHERARDI, Silvia y Nicolini, Davide. "Learning in a Constellation of Interconnected Practices: Canon or Dissonance?" Journal of Management Studies. 39:4, Junio 2002. Pg419-436

HINE, Christine. Etnografia virtual. Barcelona: Editorial UOC, 2004.

Kollock, Peter. "Las economías de la colaboración on-line. Regalos y bienes públicos en el ciberespacio". En: Smith y Kollock (editores) Comunidades en el ciberspacio, Barcelona, Editorial UOC, 2003.

LaVE, Jean. "Situating Learning in Communities of Practice". En Resnick, L. B., Levine, J.M. y Teasley, S.D. (Eds). Perspectives on socially shared cognition. Washington: American Psychological Association. 1991, pg63-82

Markham, Annette N. Life online. Researching Real Experience in Virtual Space. Walnut Creek, CA: AltaMira Press. 1998, pg61-83 
RHEINGOLD, Howard. Multitudes inteligentes. Gedisa Editorial, 2004.

RezabeK, Roger J. "Online Focus Groups: Electronic Discussions for Research". Forum: Qualitative Social Research. (1) Art 18, 2000. URN: urn:nbn:de:0114fqs0001185 [última consulta: 11/11/2012] http://www.qualitativeresearch.net/index.php/fqs/article/view/1128

Wellman, Barry and HaythoRnTHWAite, Caroline (ed). The Internet en Everyday Life. Kundli: Blackwell Publishing Ltd, 2002.

Wenger, Etienne. Comunidades de Práctica: Aprendizaje, significado e identidad. Barcelona: Paidós, 2001

Wenger, Etienne; McDermott, Richard; Zinder, William. Cultivating Communities of Practice. Boston: Harvard Business School Press, 2002.

\title{
RESUMEN
}

En el marco de los cambios en el trabajo de la sociedad actual, donde la tendencia es a que los profesionistas hagan carrera de manera independiente, nos inquieta conocer las nuevas formas de construir y desarrollar una práctica al margen de las instituciones. Los sitios de red social en Internet están ofreciendo posibilidades de conexión para estos profesionistas. Presentamos el estudio de caso de uno de estos sitios que convoca a principalmente foto-reporteros independientes que viajan. Mediante una aproximación cualitativa recuperamos y describimos las formas en las que los elementos de su práctica y de su aprendizaje en la práctica se ponen en activo y se significan en el colectivo. Encontramos diferencias entre el rol de "red social" y el de "comunidad virtual" del mismo colectivo. Desde ahí abordamos una reflexión respecto del aprendizaje y desarrollo de la profesión en el marco de este tipo de colectivos.

Palabras clave: Profesionales independientes, redes sociotécnicas, comunidades virtuales, aprendizaje en la práctica

\section{Independent photojournalists at Internet. The role of the social network and that of the virtual community.}

\begin{abstract}
As work in today's society changes, with a tendency to move towards the independent worker who develops a career in a profession, we are concerned about the new ways of building and developing a practice outside of institutions. The online social networking sites are offering connecting opportunities for these professionals. We present here a case study of one of these sites dedicated to independent photojournalists that travel. Trough a qualitative approach we observed and describe in here the ways in which their practice and continuous learning get into action in the group. We found differences in the roles of the collective as "social network" and as "virtual community". From there we share our thoughts around learning and developing a profession in these Network Society's spaces.
\end{abstract}

Keywords: independent workers, social networking, virtual communities, learning in practice

\section{RÉSUMÉ}

Comme il arrive dans d'autres activités de la vie sociale de nos jours, oú la tendance à l'établiment de la figure du travailleur independent dans un métier se fait importante, on voit les changements dans le journalisme et comment les social media en offrent des opportunités pour les professionaux. A travers une mèthode quantitative ce travail montre le travail des journalistes indépendantes, leur enseigmenet continuel et les rôles qu'ils trouvent dans la "communauté virtuelle" ou dans les "reseaux sociales". On essaie d'rendre le nouveau profil des journalists pour le connaître et le developer.

Mots clé: travaillerus indépendants, journalisme, reseaux sociauux, communautés virtuelles 